\title{
Scattering of multicomponent polymer blends
}

\author{
Henrich Frielinghaus ${ }^{1}$ iD \\ Received: 1 December 2020 / Revised: 5 February 2021 / Accepted: 7 February 2021 / Published online: 23 February 2021 \\ (C) The Author(s) 2021
}

\begin{abstract}
The random phase approximation for polymer blends was developed by H. Benoît and described small angle scattering functions as well as mean field phase boundaries. It is a pure mean field theory that loses validity close to the real phase boundaries due to strong fluctuations. However, it gives a very clear roadmap about phase diagrams and scattering functions. A simplification of the random phase approximation is discussed that comes into effect when several polymers are mixed that involve a rather low number of chemically different repeat units. Then, the correlation functions of the same repeat unit pairs can be added up in a specific way such that the overall complexity for the calculations is reduced. The scattering functions and mean field phase boundaries are discussed within this concept.
\end{abstract}

Keywords Small-angle scattering $\cdot$ Phase diagrams $\cdot$ Polymer blends $\cdot$ Random phase approximation

\section{Introduction}

The random phase approximation is a mean field approach that is often applied to polymer blends [1-3]. It yields a simplified view on the scattering function and phase diagrams including interactions that neglects strong thermal composition fluctuations close to the phase transitions that can be of first or second order [4-7]. For binary homopolymer blends, it was the Flory-Huggins theory that described the mean field binodal and spinodal in a rather simple manner [8-12]. For this, entropic contributions were identified to arise from the translational entropy of placing polymers in a volume, and enthalpic contributions arise due to the monomer-monomer interactions. All of this was described on a cubic lattice. For the scattering functions [1-3], the undisturbed correlations play the role of entropy, while the interactions remain in this picture enthalpic (the independence of the scattering vector $\mathrm{Q}$ corresponds to local neighbor-neighbor interactions). Experimentally [4] and theoretically [13, 14], the interaction parameter displayed enthalpic and entropic properties that are due to monomeric structure and compressibility or free volume. However, the undisturbed correlations remained to be

Henrich Frielinghaus

h.frielinghaus@fz-juelich.de

1 Jülich Centre for Neutron Science (JCNS), Forschungszentrum Jülich GmbH, Outstation at FRM II, Lichtenbergstr. 1, 85747 Garching, Germany fully understood on the basis of the polymer architecture. For diblock copolymer blends, it was L. Leibler who introduced the mean field treatment and who predicted a phase diagram and a scattering function [15]. The strength of the mean field approach is that it can clearly give ideas about the scattering pattern and the phase diagram in a simplified way but for rather complicated polymer architectures and mixtures of them [16]. In this way, a simplified road map of a polymeric system is obtained that then could be refined by including strong fluctuations, either experimentally and/or theoretically. So for A/B/AB homopolymer/diblock copolymer blends, the group around $\mathrm{F}$. Bates studies extensively the phase diagrams and scattering $[17,18]$. Polymeric systems with many components are nowadays still highly interesting in the field of organic solar cells [19].

\section{Theory}

Let us consider $P$ different polymers, being different either in architecture or in the making by chemically different monomers. I call $N_{\mathrm{i}}$ the degree of polymerization (number of repeat units) that enters in the molecular volume of the polymers $V_{\mathrm{i}} \propto$ $N_{i}$ and the radius of gyration $R_{\mathrm{g}, i} \propto \sqrt{ } N_{i}(i=1 . . \mathrm{P})$. I call $M_{i}$ the number of chemically different repeat unit species (monomers) of polymer species $i$ (to some extent the number $M_{i}$ could be the number of blocks, but in the later example of an ABA triblock copolymer $M_{i}=2$ because I did not 
distinguish the A repeat units of the two blocks.) The overall number of chemically different repeat unit species of the whole set of polymers I call $M$.

The well-known formula of Benoit [1-3] is derived from a mean field approach applied to the scattering of polymers (polymer blends). It reads:

$\boldsymbol{S}=\left(\boldsymbol{S}_{0}^{-1}+\boldsymbol{V}\right)^{-1}$

where $\boldsymbol{S}$ is the correlation matrix of the blended polymers, $\boldsymbol{S}_{0}$ the correlation matrix of undisturbed polymers, and $\boldsymbol{V}$ the interaction matrix. Each row and column stands for a distinguishable repeat unit species (monomer) of a specific component (distinguishable polymer) that in principle can all be distinguished even if they are chemically identical (and then the interactions and scattering lengths will be the same in the respective positions of the matrices). So a system with $P$ components (i.e. different polymers), where each polymer has $M_{i}$ chemically different repeat unit species, would lead to a total dimensionality of $\Sigma M_{i}$ for Eq. (1). I now want to extend the matrices in this sense that each single polymer can in principle carry correlations between all occurring chemically different repeat unit species, but the chemically same repeat unit species-even between different polymers-shall then be treated equally. The dimensionality of Eq. (1) becomes then PM (with $M=\max M_{i}$ in most cases). So there are undisturbed correlation matrices $\boldsymbol{s}_{0, i}$ that have the dimension from all chemically distinguishable repeat unit species. If the repeat units of a particular species do not appear in this component $i$, the corresponding entries are zero (or a small $\delta$ ). On the higher level, $\boldsymbol{S}_{0}$ is now a "diagonal" matrix with the entries of the $\boldsymbol{s}_{0, i}$ matrices:

$S_{0}=\left(\begin{array}{cccc}s_{0,1} & 0 & \cdots & 0 \\ 0 & s_{0,2} & \ddots & \vdots \\ \vdots & \ddots & \ddots & 0 \\ 0 & \cdots & 0 & s_{0, N}\end{array}\right)$

where each polymer $i$ is treated separately $(i=1 . . P)$, while the interaction matrix now looks like:

$$
\begin{aligned}
V & =\left(\begin{array}{ccc}
v & \cdots & v \\
\vdots & \ddots & \vdots \\
v & \cdots & v
\end{array}\right)=\left(\begin{array}{c}
1 \\
\vdots \\
1
\end{array}\right)\left(\begin{array}{lll}
1 & \cdots & 1
\end{array}\right) \otimes v \\
& =\overrightarrow{1} \overrightarrow{1}^{\mathrm{tr}} \otimes v
\end{aligned}
$$

where the latter expression is to be understood in the bracket writing that is frequently used in quantum mechanics (dyadic product). Let us call the vector $\overrightarrow{\mathbf{1}}$ that I do not normalize. All matrices $\boldsymbol{S}_{0}, \boldsymbol{V}, \boldsymbol{s}_{0, \mathrm{i}}$ and $\boldsymbol{v}$ are Hermitian by definition. For a given vector of scattering length densities $\vec{b}$, I would obtain the final scattering function $\mathrm{S}(\mathrm{Q})$ :

$S(Q)=(\overrightarrow{\mathbf{1}} \otimes \vec{b})^{\operatorname{tr}}\left(\boldsymbol{S}_{0}^{-1}+\boldsymbol{V}\right)^{-1}(\overrightarrow{\mathbf{1}} \otimes \vec{b})$

From that I can expand the expression for large interactions $v \rightarrow \infty$ (incompressibility):

$S(Q)=(\overrightarrow{\mathbf{1}} \otimes \vec{b})^{\operatorname{tr}}\left(\frac{1}{\boldsymbol{V}}-\frac{1}{\boldsymbol{V} \boldsymbol{S}_{0} \boldsymbol{V}}+\frac{1}{\boldsymbol{V} \boldsymbol{S}_{0} \boldsymbol{V} \boldsymbol{S}_{0} \boldsymbol{V}}-\cdots\right)(\overrightarrow{\mathbf{1}} \otimes \vec{b})$

From the bracket writing of the interaction matrix, I then obtain:

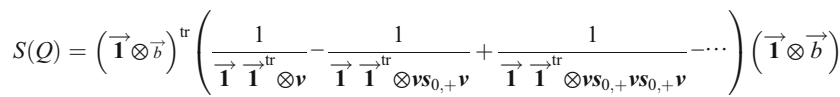

with

$\boldsymbol{s}_{0,+}=\sum \boldsymbol{s}_{0, i}$

For the reciprocal matrix of $\boldsymbol{V}$, i.e. the first term, I obtain:

$$
\begin{aligned}
(\overrightarrow{\mathbf{1}} \otimes \vec{b})^{\operatorname{tr}} \frac{1}{\boldsymbol{V}}(\overrightarrow{\mathbf{1}} \otimes \vec{b}) \\
\quad=(\overrightarrow{\mathbf{1}} \otimes \vec{b})^{\mathrm{tr}}\left(\frac{1}{P^{2}} \overrightarrow{\mathbf{1}} \overrightarrow{\mathbf{1}}^{\mathrm{tr}} \otimes \boldsymbol{v}^{-\mathbf{1}}+\sum \overrightarrow{\boldsymbol{r}}_{\boldsymbol{i}} \overrightarrow{\boldsymbol{r}}_{\boldsymbol{i}}^{\mathrm{tr}} \otimes \varepsilon_{\boldsymbol{i}}^{-\mathbf{1}}\right)(\overrightarrow{\mathbf{1}} \otimes \vec{b}) \\
=\vec{b}^{\mathrm{tr}} \boldsymbol{v}^{-\mathbf{1}} \vec{b}
\end{aligned}
$$

The normalization by the number of polymer components $P$ is due to the fact that the vector $\overrightarrow{\mathbf{1}}$ remains to be normalized. The vectors $\overrightarrow{\boldsymbol{r}}_{\boldsymbol{i}}$ are normalized and orthogonal to $\overrightarrow{\mathbf{1}}$, and they altogether span the full vector space. The rather large contributions deviating from the vector $\overrightarrow{\mathbf{1}}$ and involving $\varepsilon_{i}$ become zero in the projection. Now applying this technique to all terms, the final expression is

$S(Q)=\vec{b}^{\mathrm{tr}}\left(s_{0,+}^{-1}+v\right)^{-1} \vec{b}$

This simplification becomes evident if the different polymers include many chemically identical repeat units such that they are not distinguished in the final expression anymore. The dimensionality of Eq. 9 is now M. A simplification is reached when $M<\Sigma M_{i}$. 


\section{Examples}

One example could be a mixture of a homopolymer A, an AB diblock copolymer and a symmetric (or telechelic) ABA triblock copolymer that all consist of repeat units of type A and/ or B. Then, the final expression looks like [20]:

$\boldsymbol{s}_{0,+}=\boldsymbol{s}_{01}+\boldsymbol{s}_{02}+\boldsymbol{s}_{03}$

with the undisturbed correlation matrices:

$s_{01}=\phi_{1} V_{1}\left(\begin{array}{cc}g_{1} & 0 \\ 0 & 0\end{array}\right)$

$s_{02}=\phi_{2} V_{2}\left(\begin{array}{cc}g_{f_{2}} & \frac{1}{2}\left(g_{1}-g_{f_{2}}-g_{1-f_{2}}\right) \\ \frac{1}{2}\left(g_{1}-g_{f_{2}}-g_{1-f_{2}}\right) & g_{1-f_{2}}\end{array}\right)$

$s_{03}=\phi_{3} V_{3}\left(\begin{array}{cc}2 g_{f_{3}}+g_{1}+g_{1-2 f_{3}}-2 g_{1-f_{3}} & g_{1-f_{3}}-g_{f_{3}}-g_{1-2 f_{3}} \\ g_{1-f_{3}}-g_{f_{3}}-g_{1-2 f_{3}} & g_{1-2 f_{3}}\end{array}\right)$

with the volume fractions $\phi_{i}$ and the molar volumes $V_{i}$ of all polymers and the modified Debye functions $g_{f}(x)=\frac{2}{x^{2}}$ $(\exp (-f x)-1+f x)$ with $x=Q^{2} R_{\mathrm{g}, i}^{2}$. The magnitude $Q$ is the scattering vector, and $R_{\mathrm{g}, i}$ is the radius of gyration of the whole respective polymer. The fractions of block $\mathrm{A}$ in the three polymers are $1, f_{2}$ and $f_{3}$, where the latter measures the single end-block that occurs at either end and thus must be smaller than $1 / 2$. Equation (12 and (13 can be obtained by shifting the integration limits of the correlation functions, and so all expressions are sums of Debye functions. This polymeric system was motivated in a study of a bad batch of diblock copolymers, where on the one hand homopolymers were left from an incomplete functionalization for the second block and triblock copolymers occurred due to the presence of oxygen in the termination such that two B-blocks were linked together [20]. For simplicity, I can assume $f_{2}=1 / 2$ and $f_{3}=1 / 4$. In this two-dimensional case (with only repeat units of type A and B), the additional simplification can be made as a result of incompressibility ( $v_{\mathrm{AA}}, v_{\mathrm{BB}}, v_{\mathrm{AB}}$ large and negative):

$S(Q)=\frac{\Delta \rho^{2}}{\frac{\operatorname{sum} \boldsymbol{s}_{0,+}}{\operatorname{det} \boldsymbol{s}_{0,+}}-2 \chi}$

with the sum over all matrix elements in sum $s_{0,+}$ and the interaction parameter $\chi=\frac{\operatorname{det} v}{2 \bar{v}_{i, j}}=\frac{1}{2}\left(2 v_{A, B}-v_{A, A}-v_{B, B}\right)$ and the contrast $\Delta \rho^{2}$. The whole formula is valid for large matrix elements $v_{i, j}$. In linear chains, the sum over all matrix elements in $\boldsymbol{s}_{0,+}$ is connected to whole chain scattering $\sim g_{1}$. This formula has been found many times in the literature [3, 21].

For clarity, I calculated the scattering functions for the three materials and their mixtures. The molar volumes of an $\mathrm{A}$ and/or B block were assumed to be $20.000 \mathrm{~cm}^{3} / \mathrm{mol}$ (only in the triblock the B-block was double this size). The $f$-values were $f_{2}=1 / 2$ and $f_{3}=1 / 4$. The radii of gyration were connected to values for poly butylene oxide [22] as an example $\left(\mathrm{R}_{\mathrm{g} 1}=32 \AA, \mathrm{R}_{\mathrm{g} 2}=45 \AA\right.$, $\mathrm{R}_{\mathrm{g} 3}=63 \AA$ ), and the compositions were $\phi_{1}=0.1, \phi_{2}=0.8$ and $\phi_{3}=0.1$. We can see the scattering functions in Fig. 1. All scattering is obtained by dividing by the contrast $\Delta \rho$. For the homopolymer, I multiplied by the composition $\phi_{1}=0.1$. Otherwise for the pure components, I assumed the compositions $\phi_{i}=1$. We can see that the peak of the mixture lies between the diblock and triblock copolymer, and the forward scattering is elevated due to the presence of homopolymer (A composition can fluctuate at long length scales), while the block copolymers display the correlation hole at long length scales (no fluctuations at long length scales due to the covalent bond between $\mathrm{A}$ and $\mathrm{B}$ ).

In the next example, the interaction parameter is $\chi=0.0001$ $\mathrm{mol} / \mathrm{cm}^{3}$ (see Fig. 2). Furthermore, the original formulation with extended dimensions (Eqs. (1)-(3), see Appendix (16)-(23)) is tested against the final formulation (Eqs. (10)-(14)) with a transition of compressibility, i.e. $v_{\mathrm{AA}}=v_{\mathrm{BB}}=0.001$ over $0.01 \mathrm{~mol} /$ $\mathrm{cm}^{3}$ to $\infty$ and the respective $v_{\mathrm{AB}}=v_{\mathrm{AA}}+\chi$. For a rather compressible system (stage 1), a nearly constant contribution is added to the scattering of the incompressible system. This additive contribution becomes smaller for a less compressible system (stage 2). Finally, the incompressible system does not show any numerically significant differences between the different formulations of Eqs. (16)-(18) (Appendix) vs. Eq. (14). So, the numerical calculations also prove the validity of Eq. (14) with reduced dimensions in the limit of incompressibility.

The diblock copolymer that the model was developed for was a polybutadiene-polyisoprene (Fig. 3) with impurities [20]. The model could easily be adapted by changing the homopolymer fraction to $30 \%$ and the diblock copolymer

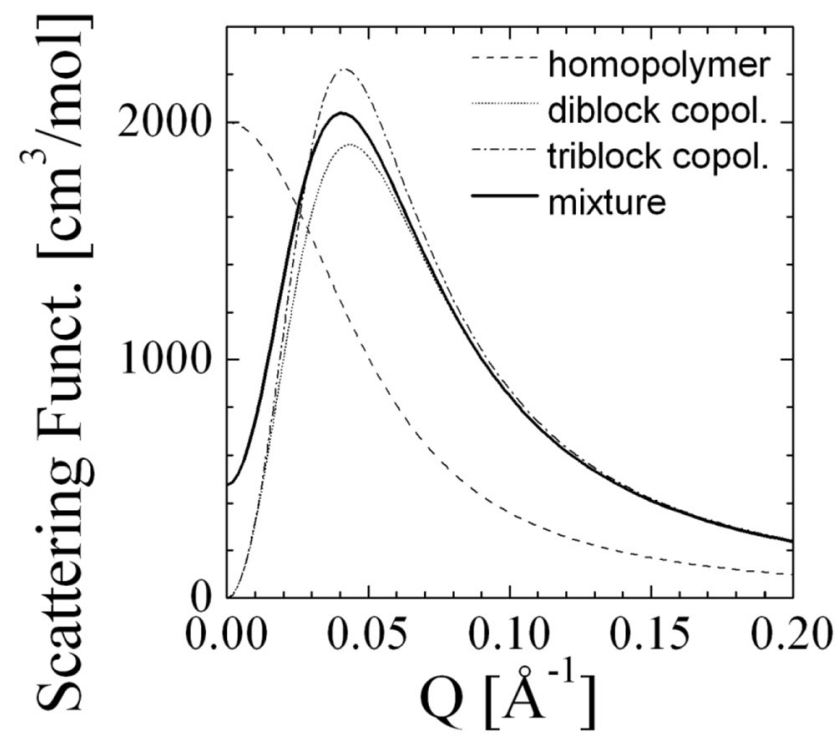

Fig. 1 The scattering functions of a homopolymer, a diblock copolymer and a triblock copolymer and the resulting one of a mixture, all with no preferable interaction, i.e. $\chi=0$ 


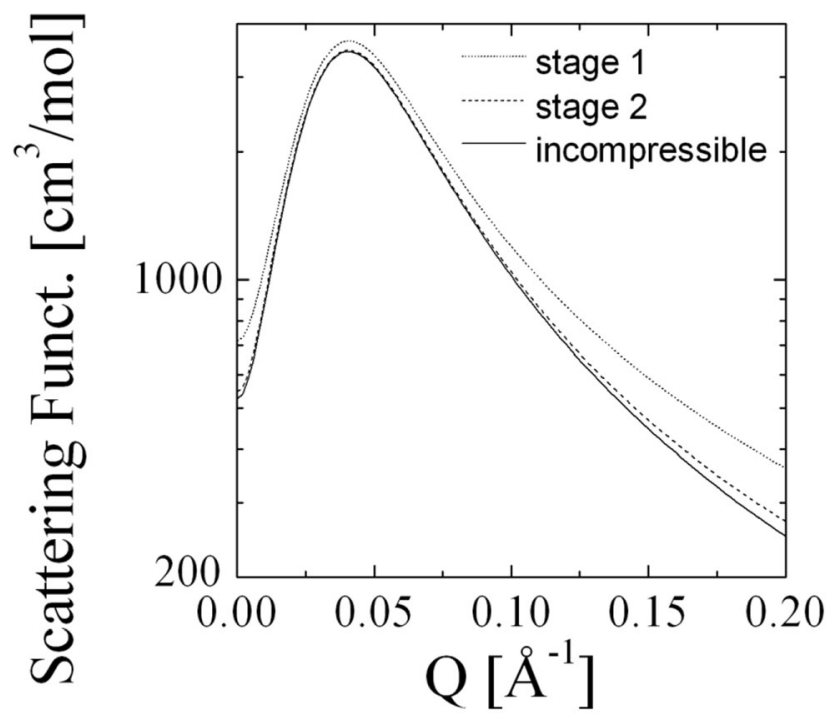

Fig. 2 The scattering function of a mixture of an A-homopolymer, an AB diblock copolymer and an A-B-A triblock copolymer (see text) with a finite interaction parameter $\chi=0.0001 \mathrm{~mol} / \mathrm{cm}^{3}$. Additionally, the monomer-monomer interactions are rendered from $v_{\mathrm{AA}}=v_{\mathrm{BB}}=0.001$ (stage 1) over $0.01 \mathrm{~mol} / \mathrm{cm}^{3}$ (stage 2) to $\infty$ (and the respective $v_{\mathrm{AB}}=v_{\mathrm{AA}}+$ $\chi$ ) to simulate different states of compressibility

fraction to $60 \%$. The radii of gyration were multiplied by 5 , the interaction parameter was $\chi=0.000047 \mathrm{~mol} / \mathrm{cm}^{3}$, and the molar volume was $3.7 \times 10^{5} \mathrm{~cm}^{3} / \mathrm{mol}$. As one can see, the model describes the scattering very well. The high amount of homopolymer is in the range measured by a GPC/SEC trace. However, the polydispersity of the block fraction $f$ could also contribute to the high scattering level at lowest $Q$. We discuss polydispersity further below.

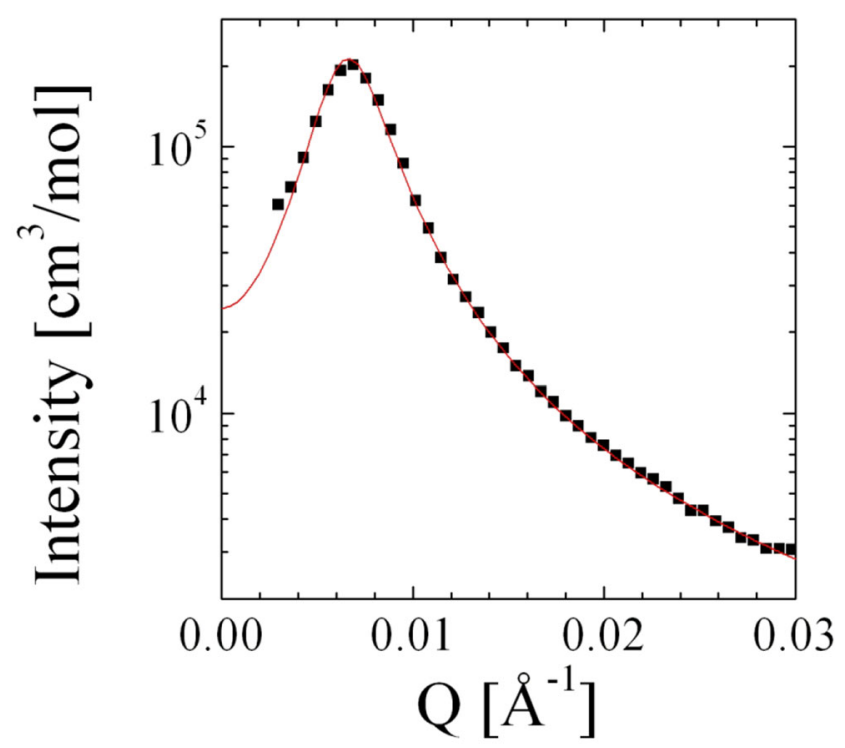

Fig. 3 The measured polybutadiene-polyisoprene (d-PB(1,4)-PI) diblock copolymer at $130^{\circ} \mathrm{C}$ with a molar mass of $350 \mathrm{~kg} / \mathrm{mol}$ as described with the model. The homopolymer fraction was $30 \%$. The interaction parameter was $\chi=0.000047 \mathrm{~mol} / \mathrm{cm}^{3}$. The set of radii of gyration was 5 times larger
Generally, mean field phase diagrams, i.e. the spinodals, can be obtained by finding the zeros of the determinant:

$\operatorname{det}\left(\boldsymbol{s}_{0,+}^{-1}+\boldsymbol{v}\right)=0$

By the analysis, which vector leads to the zero of the determinant, one can distinguish the type of phase separation [16, 23]. An example was the blend of an $\mathrm{AB}$ and an $\mathrm{BC}$ diblock copolymer, where the indices follow the order $\mathrm{A}, \mathrm{B}, \mathrm{C}$. Then, the vector $(1,0,-1) / \sqrt{2}$ at the critical scattering vector $Q^{*}=0$ indicates macrophase separation, and another vector including components of $(1,2,1) / \sqrt{ } 6$ at a critical scattering vector $Q^{*}>0$ indicates microphase separation. The periodicity of the phase-separated domains is given by $d=2 \pi / Q^{*}$. When the two conditions merge at a single point, the Lifshitz critical point is obtained. Here, the macrophase and microphase separations are indistinguishable. However, in the practical examples, this behavior has not been observed [19]. Another example for a Lifshitz criticality is the mixture of $\mathrm{A} / \mathrm{B}$ homopolymers with $\mathrm{AB}$ diblock copolymers [24, 25]. This criticality leads to a formation of bicontinuous microemulsion domain structures that extend to quite low temperatures. It has to be mentioned that the quite severe extension of the microemulsion phase is due to the presence of strong fluctuations that are neglected by the mean field approach discussed here.

In the example system, for which I calculated the scattering functions, all matrices become 2-dimensional, and a further simplification applies: The denominator of Eq. 14 must become zero at the mean field phase boundary, and so values of the interaction parameter $\chi$ can be calculated for the $Q$-value zero and at the minimum $Q^{*}$ of sum $\boldsymbol{s}_{0,+} / \operatorname{det} \boldsymbol{s}_{0,+}$. The two phase boundaries and the values of $Q^{*}$ are depicted in Fig. 4. The phase boundary connected to $Q=0$ is the spinodal of the macrophase separation and the other one the order-disorder phase transition (i.e. microphase transition). As a system, we

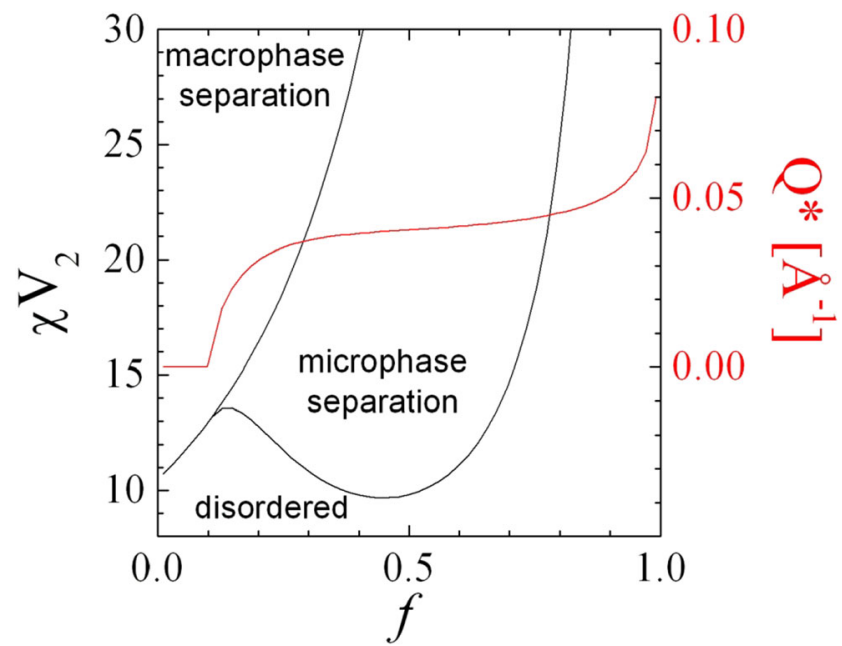

Fig. 4 Phase diagram of an A-homopolymer, an A-B diblock copolymer and an A-B-A triblock copolymer (see text). The molar volume of the Ahomopolymer of $20 ., 000 \mathrm{~cm}^{3} / \mathrm{mol}$ is constant while the molar volumes of the A-blocks vary according to the $f$-value, i.e. $f_{2}=f$ and $f_{3}=f / 2$. 
assumed that the A homopolymer has a constant molar volume of $\mathrm{V}_{1}=20 ., 000 \mathrm{~cm}^{3} / \mathrm{mol}$, while the A block molar volumes vary with the $f$-value $\left(f_{3}=f_{2} / 2\right)$.

It should be stressed that the equations above hold for onephase disordered systems. So, if the phase boundary is crossed, the approach is invalid and does not predict the scattering function anymore. Good comparisons between calculations and experiments can be found in [21].

The simplification of reduced dimensions as expressed in Eqs. (9) and (15) was already implicitly used for polydisperse systems [26, 27], where one simply argues that the different components due to the polydispersity are such similar that this simplified approach is valid. However, I argue that even very different polymers can lead to the simplified expressions as mentioned in this paper.

\section{Conclusions}

The final theoretical result of this paper is given in Eqs. (7) and (9). It reduces the unnecessary dimensions of the correlations that may arise if chemically same repeat units on different polymers are distinguished. I presented a calculation on how these dimensions can be minimized, although the intermediate matrices are expanded to dimension $n \times n=(P M) \times(P M)$, with $P$ being the number of different polymers and $M$ being the overall number of all chemically different repeat units involved. The final formula reduces the matrices to a dimension $M \times M$. An example for a blend of an A homopolymer, an AB diblock copolymer and a symmetric ABA triblock copolymer is discussed explicitly. We displayed scattering functions and a mean field phase diagram. Other examples of $\mathrm{AB}$ and $\mathrm{BC}$ diblock copolymer blends and A/B homopolymer blends with $\mathrm{AB}$ diblock copolymers are discussed peripherally and are referred to the cited literature.

Acknowledgements I thank Hirokazu Hasegawa for providing his first batch of the diblock copolymer and consulting about its characterization. I thank Dietmar Schwahn for supervising my diploma thesis at that time.

Funding Open Access funding enabled and organized by Projekt DEAL.

\section{Declarations}

Conflict of interest The authors declare that they have no conflict of interest.

\section{Appendix}

For the example system of the three polymers, an A homopolymer, an AB diblock copolymer and an ABA triblock copolymer (Eqs. (11)-(13)), we explicitly show the simplification. The original correlation matrix $\boldsymbol{S}_{0}$ is then:

$\boldsymbol{S}_{0}=\left(\begin{array}{cccc}\phi_{1} V_{1} g_{1} & 0 & 0 & 0 \\ 0 & \delta & 0 & 0 \\ 0 & 0 & \phi_{2} V_{2} g_{f_{2}} & \frac{1}{2} \phi_{2} V_{2}\left(g_{1}-g_{f_{2}}-g_{1-f_{2}}\right) \\ 0 & 0 & \frac{1}{2} \phi_{2} V_{2}\left(g_{1}-g_{f_{2}}-g_{1-f_{2}}\right) & \phi_{2} V_{2} g_{1-f_{2}} \\ 0 & 0 & 0 & 0 \\ 0 & 0 & 0 & 0\end{array}\right.$

$$
\left.\begin{array}{cc}
0 & 0 \\
0 & 0 \\
0 & 0 \\
0 & 0 \\
\phi_{3} V_{3}\left(2 g_{f_{3}}+g_{1}+g_{1-2 f_{3}}-2 g_{1-f_{3}}\right) & \phi_{3} V_{3}\left(g_{1-f_{3}}-g_{f_{3}}-g_{1-2 f_{3}}\right) \\
\phi_{3} V_{3}\left(g_{1-f_{3}}-g_{f_{3}}-g_{1-2 f_{3}}\right) & \phi_{3} V_{3} g_{1-2 f_{3}}
\end{array}\right)
$$

The interaction matrix reads then:

$$
\boldsymbol{V}=\left(\begin{array}{llllll}
v_{A, A} & v_{A, B} & v_{A, A} & v_{A, B} & v_{A, A} & v_{A, B} \\
v_{A, B} & v_{B, B} & v_{A, B} & v_{B, B} & v_{A, B} & v_{B, B} \\
v_{A, A} & v_{A, B} & v_{A, A} & v_{A, B} & v_{A, A} & v_{A, B} \\
v_{A, B} & v_{B, B} & v_{A, B} & v_{B, B} & v_{A, B} & v_{B, B} \\
v_{A, A} & v_{A, B} & v_{A, A} & v_{A, B} & v_{A, A} & v_{A, B} \\
v_{A, B} & v_{B, B} & v_{A, B} & v_{B, B} & v_{A, B} & v_{B, B}
\end{array}\right)
$$

The original formula to be calculated, now including the scattering length densities $b_{\mathrm{A}}$ and $b_{\mathrm{B}}$ would finally read:

$S(Q)=\left(\begin{array}{llllll}b_{A} & b_{B} & b_{A} & b_{B} & b_{A} & b_{B}\end{array}\right)\left(\boldsymbol{S}_{0}^{-1}+\boldsymbol{V}\right)^{-1}\left(\begin{array}{c}b_{A} \\ b_{B} \\ b_{A} \\ b_{B} \\ b_{A} \\ b_{B}\end{array}\right)$ 
The calculation in a computer would not face large difficulties. However, the envisaged analytical simplifications provide a better insight and errors can be found easier. So the sum of the $2 \times 2$ correlation matrices reads:

$\boldsymbol{s}_{0,+}=\left(\begin{array}{cc}\phi_{1} V_{1} g_{1}+\phi_{2} V_{2} g_{f_{2}}+\phi_{3} V_{3}\left(2 g_{f_{3}}+g_{1}+g_{1-2 f_{3}}-2 g_{1-f_{3}}\right) & \frac{1}{2} \phi_{2} V_{2}\left(g_{1}-g_{f_{2}}-g_{1-f_{2}}\right)+\phi_{3} V_{3}\left(g_{1-f_{3}}-g_{f_{3}}-g_{1-2 f_{3}}\right) \\ \frac{1}{2} \phi_{2} V_{2}\left(g_{1}-g_{f_{2}}-g_{1-f_{2}}\right)+\phi_{3} V_{3}\left(g_{1-f_{3}}-g_{f_{3}}-g_{1-2 f_{3}}\right) & \phi_{2} V_{2} g_{1-f_{2}}+\phi_{3} V_{3} g_{1-2 f_{3}}\end{array}\right)$

and the interaction matrix reads:

$\boldsymbol{v}=\left(\begin{array}{ll}v_{A, A} & v_{A, B} \\ v_{A, B} & v_{B, B}\end{array}\right)$

So, the general result in terms of a simplified Benoit theory (Eq. 9) would arrive at:

$S(Q)=\left(\begin{array}{ll}b_{A} & b_{B}\end{array}\right)\left(s_{0,+}^{-1}+v\right)^{-1}\left(\begin{array}{l}b_{A} \\ b_{B}\end{array}\right)$

The final scattering formula, assuming incompressibility, would now read (as in Eq. 14):

$S(Q)=\frac{\Delta \rho^{2}}{\frac{\operatorname{sum} \boldsymbol{s}_{0,+}}{\operatorname{det} \boldsymbol{s}_{0,+}}-2 \chi}$

Note that we call $\Delta \rho=b_{\mathrm{A}}-b_{\mathrm{B}}$. As a cross check, we obtain for the sum of all entries from $s_{0,+}$ the single chain scattering:

sum $\boldsymbol{s}_{0,+}=\phi_{1} V_{1} g_{1}+\phi_{2} V_{2} g_{1}+\phi_{3} V_{3} g_{1}$

Please note that the entries for the radii of gyration to the terms $g_{1}\left(Q R_{\mathrm{g}, \mathrm{i}}\right)$ may be different in the three addends. So the only remaining difficult term is the determinant of $\boldsymbol{s}_{0,+}$, which introduces products of correlations.

Open Access This article is licensed under a Creative Commons Attribution 4.0 International License, which permits use, sharing, adaptation, distribution and reproduction in any medium or format, as long as you give appropriate credit to the original author(s) and the source, provide a link to the Creative Commons licence, and indicate if changes were made. The images or other third party material in this article are included in the article's Creative Commons licence, unless indicated otherwise in a credit line to the material. If material is not included in the article's Creative Commons licence and your intended use is not permitted by statutory regulation or exceeds the permitted use, you will need to obtain permission directly from the copyright holder. To view a copy of this licence, visit http://creativecommons.org/licenses/by/4.0/.

\section{References}

1. J. Higgins, Benoit HC, Polymers and neutron scattering (chapter 7), Clarendon Press, Oxford, 1994.

2. Vilgis TA, Benmouna M, Benoit H (1991) Static scattering from multicomponent polymer systems: theoretical models. Macromolecules 24:4481-4488. https://doi.org/10.1021/ ma00016a001

3. Vilgis TA (2000) Polymer theory: path integrals and scaling. Phys Rep 336:167-254. https://doi.org/10.1016/S0370-1573(99)001222

4. Schwahn D, Mortensen K, Yee-Madeira H (1987) Mean-field and Ising critical behavior of a polymer blend. Phys Rev Lett 58:1544. https://doi.org/10.1103/PhysRevLett.58.1544

5. Frielinghaus H, Schwahn D, Mortensen K, Almdal K, Springer T (1996) Composition fluctuations and coil conformation in a poly (ethylene- propylene)- poly (ethylethylene) diblock copolymer as a function of temperature and pressure. Macromolecules 29:32633271. https://doi.org/10.1021/ma950998w

6. Belyakov MY, Kiselev SB (1992) Crossover behavior of the susceptibility and the specific heat near a second-order phase transition. Phys A Stat Mech App 190:75-94. https://doi.org/10.1016/ 0378-4371(92)90078-5

7. Fredrickson GH, Helfand E (1987) Fluctuation effects in the theory of microphase separation in block copolymers. J Chem Phys 87: 697-705. https://doi.org/10.1063/1.453566

8. Flory PJ (1941) Thermodynamics of high polymer solutions. J Chem Phys 9:660-660. https://doi.org/10.1063/1.1750971

9. Flory PJ (1942) Thermodynamics of high polymer solutions. J Chem Phys 10:51-61. https://doi.org/10.1063/1.1723621

10. Huggins ML (1941) Solutions of long chain compounds. J Chem Phys 9:440-440. https://doi.org/10.1063/1.1750930

11. Huggins ML (1942) Some properties of solutions of long-chain compounds. J Phys Chem 46:151-158. https://doi.org/10.1021/ j150415a018

12. Huggins ML (1942) Theory of solutions of high polymers. J Am Chem Soc 64:1712-1719. https://doi.org/10.1021/ja01259a068

13. Dudowicz J, Freed KF (1991) Effect of monomer structure and compressibility on the properties of multicomponent polymer blends and solutions: 1. Lattice cluster theory of compressible systems. Macromolecules 24:5076-5095. https://doi.org/10.1021/ ma00018a014

14. Dudowicz J, Freed KF (1993) Relation of effective interaction parameters for binary blends and diblock copolymers: lattice cluster theory predictions and comparisons with experiment. Macromolecules 26:213-220. https://doi.org/10.1021/ ma00053a033

15. Leibler L (1980) Theory of microphase separation in block copolymers. Macromolecules 13:1602-1617. https://doi.org/10.1021/ ma60078a047 
16. Olmsted PD, Hamley IW (1999) Lifshitz points in blends of AB and BC diblock copolymers. Europhys Lett 45:83-89. https://doi. org/10.1209/epl/i1999-00135-4

17. Hillmyer MA, Maurer WW, Lodge TP, Bates FS, Almdal K (1999) Model bicontinuous microemulsions in ternary homopolymer/ block copolymer blends. J Phys Chem B 103:4814 4824. https:// doi.org/10.1021/jp990089z

18. Washburn NR, Lodge TP, Bates FS (2000) Ternary polymer blends as model surfactant systems. J Phys Chem B 104:6987-6997. https://doi.org/10.1021/jp994230f

19. Wu J, Li G, Fang J, Guo X, Zhu L, Guo B, Wang Y, Zhang G, Arunagiri L, Liu F, Yan H, Zhang M, Li Y (2020) Random terpolymer based on thiophene-thiazolothiazole unit enabling efficient non-fullerene organic solar cells. Nat Commun 11:1-9. https:// doi.org/10.1038/s41467-020-18378-9

20. H. Frielinghaus (1994). SANS-Untersuchungen der Druckabhangigkeit von Diblockcopolymer-Phasendiagrammen. Diploma thesis, Rheinisch-Westfalische Technische Hochshule Aachen.

21. Chintapalli M, Timachova K, Olson KR, Banaszak M, Thelen JL, Mecham SJ, DeSimone JM, Balsara NP (2017) Incipient microphase separation in short chain perfluoropolyether-block-poly (ethylene oxide) copolymers. Soft Matter 13:4047-4056. https://doi. org/10.1039/C7SM00738H

22. Gerstl C, Schneider GJ, Pyckhout-Hintzen W, Allgaier J, Willbold S, Hofmann D, Disko U, Frielinghaus H, Richter D (2011) Chain conformation of poly (alkylene oxide) s studied by small-angle neutron scattering. Macromolecules 44:6077-6084. https://doi. org/10.1021/ma201288a
23. Frielinghaus H, Hermsdorf N, Almdal K, Mortensen K, Messé L, Corvazier L, Fairclough JPA, Ryan AJ, Olmsted PD, Hamley IW (2001) Micro-vs. macro-phase separation in binary blends of poly (styrene)-poly (isoprene) and poly (isoprene)-poly (ethylene oxide) diblock copolymers. Europhys Lett 53:680-686. https://doi.org/10. 1209/epl/i2001-00205-7

24. Schwahn D, Mortensen K, Frielinghaus H, Almdal K (1999) Crossover from 3D ising to isotropic Lifshitz critical behavior in a mixture of a homopolymer blend and diblock copolymer. Phys Rev Lett 82:5056-5059. https://doi.org/10.1103/PhysRevLett.82. 5056

25. Bates FS, Maurer W, Lodge TP, Schulz MF, Matsen MW, Almdal K, Mortensen K (1995) Isotropic Lifshitz behavior in block copolymer-homopolymer blends. Phys Rev Lett 75:4429-4432. https://doi.org/10.1103/PhysRevLett.75.4429

26. Cooke DM, Shi AC (2006) Effects of polydispersity on phase behavior of diblock copolymers. Macromolecules 39:6661-6671. https://doi.org/10.1021/ma060717s

27. Sakurai S, Mori K, Okawara A, Kimishima K, Hashimoto T (1992) Evaluation of segmental interaction by small-angle X-ray scattering based on the random-phase approximation for asymmetric, polydisperse triblock copolymers. Macromolecules 25:2679-2691. https:// doi.org/10.1021/ma00036a019

Publisher's note Springer Nature remains neutral with regard to jurisdictional claims in published maps and institutional affiliations. 